\title{
Dispersion Characteristics of a Cylindrical Electromagnetic Band Gap Structure
}

\author{
Halim Boutayeb and Kouroch Mahdjoubi
}

\begin{abstract}
In this letter, a new analytical expression for the dispersion equation of radially periodic structures is derived. The periodic structure is considered as a set of parallel cylindrical Frequency Selective Surfaces (FSSs), and the dispersion equation is calculated by using a transmission line model. Using this result, the dispersion proprieties of Cylindrical Electromagnetic Bandgap (CEBG) structures composed of continuous or discontinuous metallic wires are presented. It is shown that the band structures of these materials can be determined by using the proposed dispersion equation. Cylindrical periodic materials have potential applications for designing directive antennas, circular high impedance surfaces or agile antennas.
\end{abstract}

Index Terms-Periodic structures, dispersion equation, cylindrical structures

\section{INTRODUCTION}

C YLINDRICAL Electromagnetic Bandgap (CEBG) structures [1] are circularly and radially periodic materials which present pass-bands and stop-bands to cylindrical electromagnetic waves. Potential applications of these structures have been proposed in microwave and antenna domains for wireless communication systems [1, 2]. In [1], analytical expressions have been proposed to obtain the reflection and transmission coefficients of a cylindrical EBG structure, and a directive antenna based on this structure has been proposed, fabricated and tested. In addition, the authors have proposed to use a cylindrical EBG structure with active elements to obtain a reconfigurable directive beam over $360^{\circ}$ range. In [2], a cylindrical EBG-based antenna with a high directivity in the elevation plane and wide horizontal beam has been presented. It is well known that the propagation of waves in periodic structures is described by means of a band theory. For instance, the dispersion proprieties of crystals with a rectangular lattice is often used for the design of microwave and optical components [3]. However, cylindrical periodic materials are not often used, probably because their properties have not been analyzed enough. In this letter, a new analytical expression for the dispersion equation of a radially periodic structure is derived. The periodic structure is considered as a set of parallel cylindrical Frequency Selective Surfaces (FSSs). Using the proposed dispersion equation, the pass-bands and stop-bands of cylindrical EBG structures composed of continuous or discontinuous metallic wires are predicted. It is shown that the predicted results are in good agreement with the band structure deduced from the transmission coefficients.

Manuscript received April 23, 2006; Revised July 13, 2006.

H. Boutayeb is with INRS-EMT, 800 de la Gauchetiere O., Montréal, H5A 1K6, Canada, E-mail: boutayeb@emt.inrs.ca.

K. Mahdjoubi is from IETR, Av. du Gal Leclerc, 35042 Rennes, France.

\section{Characterization of A RAdially Periodic STRUCTURE}

In this section, the dispersion equation of radially periodic structures composed of multiple layers of cylindrical Frequency Selective Surfaces (FSSs) is derived by using a transmission line model, where a cylindrical FSS is modelled by a T circuit.

\section{A. Transmission line for a cylindrical wave}

The propagation of the cylindrical waves in the line section of width $P$ as illustrated in Fig. 1 is considered.

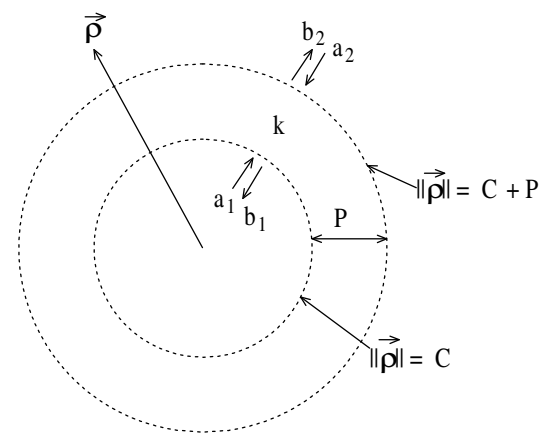

Fig. 1. Characterization of the transmission line of width $P$, for a cylindrical wave in free space. The line starts at $\|\rho\|=C$ and ends at $\|\rho\|=C+P$.

The relationships between the powers $a_{1}, b_{1}, a_{2}$ and $b_{2}$ shown in Fig. 1 can be written

$$
b_{1}=e^{\eta_{0}(k C)-\eta_{0}(k(C+P))} a_{2}
$$

and

$$
b_{2}=e^{\eta_{0}(k C)-\eta_{0}(k(C+P))} a_{1}
$$

where $k$ is the free space wave number and $\eta_{0}(x)$ is the phase of the cylindrical wave:

$$
\eta_{0}(x)=\arctan \left(\frac{N_{0}(x)}{J_{0}(x)}\right)
$$

where $J_{0}(x)$ and $N_{0}(x)$ are the first and second kind Bessel functions of zero order.

Then, the $\mathrm{S}$ matrix of the transmission line section can be written

$$
\left(\begin{array}{cc}
0 & e^{\eta_{0}(k C)-\eta_{0}(k(C+P))} \\
e^{\eta_{0}(k C)-\eta_{0}(k(C+P))} & 0
\end{array}\right)
$$

From the $\mathrm{S}$ matrix, the chain matrix of the line section is

$$
\left(\begin{array}{cc}
\cos (A) & j \sin (A) \\
j \sin (A) & \cos (A)
\end{array}\right)
$$


where

$$
A=\eta_{0}(k(C+P))-\eta_{0}(k C)
$$

\section{B. Circuit model of a cylindrical FSS}

A cylindrical FSS as shown in Fig. 2(a) is considered. This structure is characterized by its transmission and reflection coefficients, $t$ and $r$. Figure 2(b) presents the $\mathrm{T}$ circuit model of the cylindrical shell.

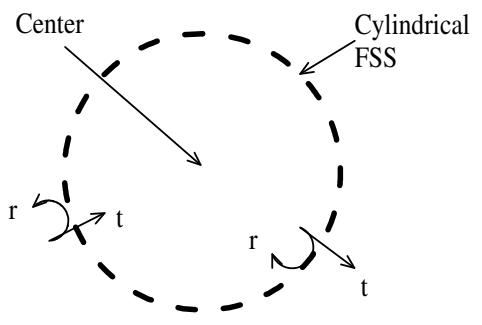

(a)



(b)
Fig. 2. (a) Characteristics of a Cylindrical Frequency Selective Surface. (b) $\mathrm{T}$ circuit model of the cylindrical shell.

The chain matrix of the $\mathrm{T}$ circuit is written

$$
\left(\begin{array}{cc}
b & c \\
d & b
\end{array}\right)=\left(\begin{array}{cc}
1+Y Z & 2 Z+Y Z^{2} \\
Y & 1+Y Z
\end{array}\right)
$$

where the terms $Z$ and $Y$ are written as functions of $r$ and $t$

$$
\begin{gathered}
Y=\frac{(r-t-1)(r+t-1)}{2 t} \\
Z=\frac{-r+t-1}{r-t-1}
\end{gathered}
$$

\section{Multilayered cylindrical structures}

A radially periodic structure as shown in Fig. 3 is now considered. The structure is composed of multiple layers of cylindrical FSSs, which are periodically spaced with the radial period $P_{r}$ and have the same coefficients $t$ and $r$. The transmission line model of the periodic structure is presented in Fig. 4. An elementary cell is composed of the cylindrical shell and of two transmission lines of length $P_{r} / 2$ on each side of the shell. Using the previous results, the chain matrixes of these two sections are given by :

$$
\left(\begin{array}{cc}
\alpha_{1} & \gamma_{1} \\
\gamma_{1} & \alpha_{1}
\end{array}\right)=\left(\begin{array}{cc}
\cos \left(A_{1}\right) & j \sin \left(A_{1}\right) \\
j \sin \left(A_{1}\right) & \cos \left(A_{1}\right)
\end{array}\right)
$$

and

$$
\left(\begin{array}{ll}
\alpha_{2} & \gamma_{2} \\
\gamma_{2} & \alpha_{2}
\end{array}\right)=\left(\begin{array}{cc}
\cos \left(A_{2}\right) & j \sin \left(A_{2}\right) \\
j \sin \left(A_{2}\right) & \cos \left(A_{2}\right)
\end{array}\right)
$$

where

$$
A_{1}=\eta_{0}\left(k P_{r}\right)-\eta_{0}\left(k \frac{P_{r}}{2}\right)
$$

and

$$
A_{2}=\eta_{0}\left(k \frac{3 P_{r}}{2}\right)-\eta_{0}\left(k P_{r}\right)
$$

The chain matrix of the elementary cell is then obtained by multiplying the different chain matrixes:

$$
\left(\begin{array}{cc}
\alpha_{1} & \gamma_{1} \\
\gamma_{1} & \alpha_{1}
\end{array}\right)\left(\begin{array}{ll}
b & c \\
d & b
\end{array}\right)\left(\begin{array}{ll}
\alpha_{2} & \gamma_{2} \\
\gamma_{2} & \alpha_{2}
\end{array}\right)
$$

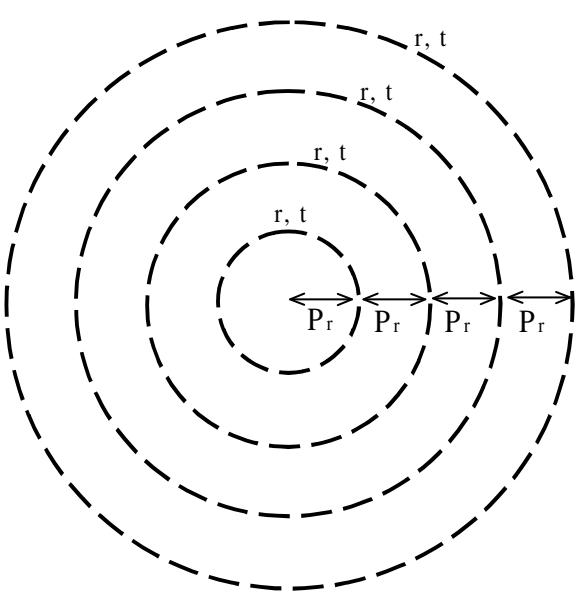

Fig. 3. Radially periodic structure.

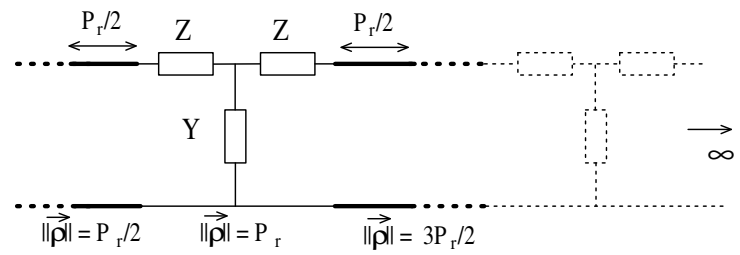

Fig. 4. Transmission line model of the periodic structure in Fig. 3.

This chain matrix can also be written

$$
\left(\begin{array}{cc}
\cos (B) & j \sin (B) \\
j \sin (B) & \cos (B)
\end{array}\right)
$$

where

$$
B=\eta_{0}\left(\beta \frac{3 P_{r}}{2}\right)-\eta_{0}\left(\beta \frac{P_{r}}{2}\right)
$$

and $\beta$ is the propagation constant of the transmission line. By identifying the first terms of the two matrixes given by Eqs. 14 and 15, we obtain the dispersion equation of the structure

$$
\begin{aligned}
& \cos \left(\eta_{0}\left(\beta \frac{3 P_{r}}{2}\right)-\eta_{0}\left(\beta \frac{P_{r}}{2}\right)\right)=(1+Y Z) \cos \left(A_{3}\right) \\
& +j\left(2 Z+Y Z^{2}\right) \cos \left(A_{1}\right) \sin \left(A_{2}\right) \\
& +j Y \cos \left(A_{2}\right) \sin \left(A_{1}\right)
\end{aligned}
$$

where

$$
A_{3}=A_{1}+A_{2}=\eta_{0}\left(k \frac{3 P_{r}}{2}\right)-\eta_{0}\left(k \frac{P_{r}}{2}\right)
$$

Eq. 17 is an extension to the cylindrical case of the formula for multi-sections transmission lines [4]. The coefficient $\eta_{0}\left(\beta \frac{3 P_{r}}{2}\right)-\eta_{0}\left(\beta \frac{P_{r}}{2}\right)$ has real values in pass-bands. Whereas, it has no real value in stop-bands. Then, the band structure of the cylindrical periodic material can be obtained by using Eq. 17 if one knows the transmission and reflection coefficients $t$ and $r$ of one cylindrical shell.

\section{RESUltS}

Now, we consider a Cylindrical EBG structure composed of metallic wires as shown in Fig. 5. The propagation of the 
transverse Electric field in the radial direction is considered. The cylindrical surfaces are periodically spaced with the period $P_{r}$ and have the same transversal period $\left(P_{t}\right)$, and accordingly the same transmission and reflection coefficients $(t$ and $r)$ [1]. According to [1], the transmission and reflection coefficients of this CEBG structure with $n$ cylindrical shells can be written

$$
\begin{gathered}
t_{n}=\frac{t_{n-1} t}{1-r_{n-1} r e^{\left.-j 2 \eta_{0}\left(k n P_{r}\right)\right)+j 2 \eta_{0}\left(k(n-1) P_{r}\right)}} \\
r_{n}=r_{n-1}+\frac{t_{n-1}^{2} r e^{\left.-j 2 \eta_{0}\left(k n P_{r}\right)\right)+j 2 \eta_{0}\left(k P_{r}\right)}}{1-r_{n-1} r e^{\left.-j 2 \eta_{0}\left(k n P_{r}\right)\right)+j 2 \eta_{0}\left(k(n-1) P_{r}\right)}}
\end{gathered}
$$

where $t_{1}=t$ and $r_{1}=r$.

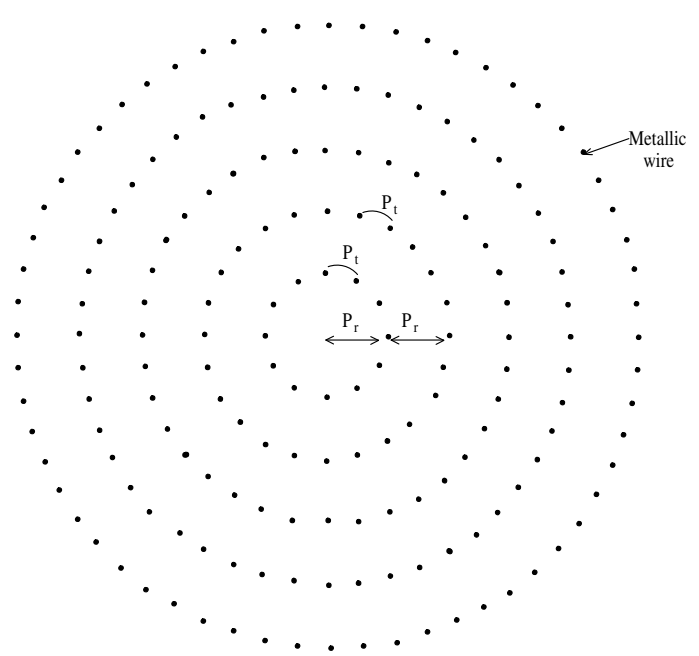

Fig. 5. CEBG structure composed of metallic wires. $P_{r}=45 \mathrm{~mm}, P_{t}=$ $45 \times \pi / 6 \mathrm{~mm}$ and the wire diameter is $2 \mathrm{~mm}$.
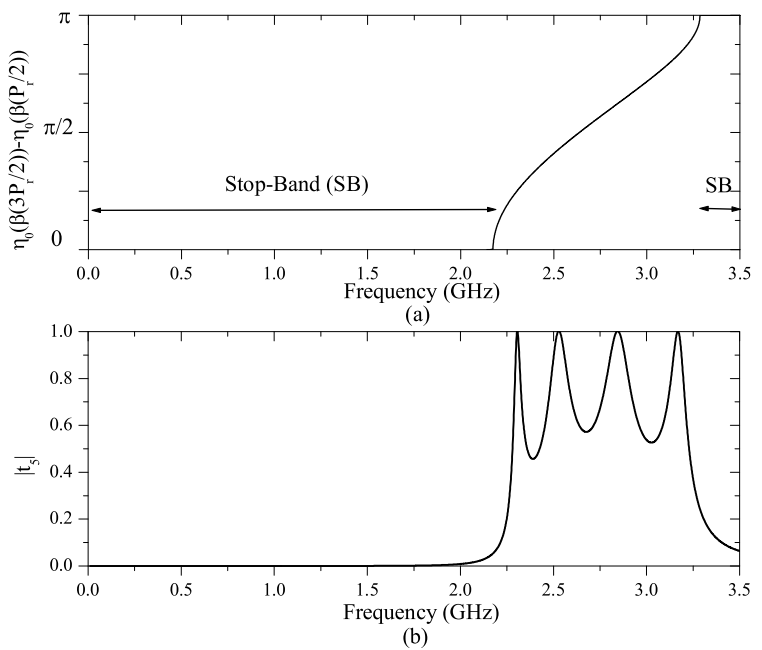

Fig. 6. (a) coefficient $\eta_{0}\left(\beta \frac{3 P_{r}}{2}\right)-\eta_{0}\left(\beta \frac{P_{r}}{2}\right)$ (Eq. (17) ) and (b) coefficient $\left|t_{5}\right|$ (Eqs. (19) and (20)) of a CEBG structure with continuous metallic wires.

The coefficients $t$ and $r$ of a cylindrical shell were calculated as follows: first, the total fields inside and outside the cylindrical cavity, excited by a line source in its center, were calculated by using a Finite Difference Time Domain code; Then, the coefficients $t$ and $r$ were extracted by considering that multiple reflections occur between the surface and the center [1]. Using these results, the coefficient $\eta_{0}\left(\beta \frac{3 P_{r}}{2}\right)-\eta_{0}\left(\beta \frac{P_{r}}{2}\right)$ and the transmission coefficient $\left|t_{5}\right|$ of a structure with continuous metallic wires were calculated and are plotted in Fig. 6. From Fig. 6, the band structures given by the transmission coefficient and the dispersion equation are in good agreement. Fig. 7 presents the same diagrams as that of Fig. 6 for a structure composed of discontinuous wires. The finite wires are $18 \mathrm{~mm}$ length and the vertical distance between two wires is $2 \mathrm{~mm}$. The radial period and the wire diameter are the same than previously. From Fig. 7, the proposed dispersion equation gives also a good prediction of the band structure of the cylindrical periodic material.


Fig. 7. coefficients (a) $\eta_{0}\left(\beta \frac{3 P_{r}}{2}\right)-\eta_{0}\left(\beta \frac{P_{r}}{2}\right)$ (Eq. (17)) and (b) $\left|t_{5}\right|$ (Eqs. (19) and (20)) of a CEBG structure with discontinuous metallic wires.

\section{CONCLUSION}

A new analytical expression for the characterization of cylindrical periodic materials has been presented. The dispersion equation has been calculated for determining the band structure of these materials. The obtained results are in good agreement with the band structure deduced from the transmission coefficients. Due to their angular isotropy, cylindrical periodic structures have potential applications in antenna and microwave domains such as circular high impedance surfaces, agile antennas or circular reflectors.

\section{REFERENCES}

[1] H. Boutayeb, T.A. Denidni, K. Mahdjoubi, A.-C. Tarot, A. Sebak and L.Talbi, "Analysis and Design of a Cylindrical EBG-based directive antenna, IEEE Trans. Ant. Propag., vol.54, pp. 211-219, 2006.

[2] G.K. Palikaras, A.P. Feresidis, and J.C. Vardaxoglou, "Cylindrical Electromagnetic bandgap structures for direcive Base Station Antennas", IEEE Ant. Wir. Propagat. Lett., vol. 3, pp. 87-89, 2004.

[3] K. Sakoda, Optical Properties of Photonic Crystal, ser. Opt. Sci. Berlin, Germany: Springer, 2001, vol. 80.

[4] R.E. Collin, Foundations for Microwave Engineering - Second Edition, Chap. 5, McGraw-Hill International Editions, 1992. 